\title{
Periodismo argentino: en busca de datos sobre la profesión
}

Adriana Amado Suárez

Universidad Nacional de La Matanza, San Justo, prov. de Buenos Aires

\section{Resumen}

Argentina tiene una larga tradición en el periodismo, con instituciones académicas pioneras en la enseñanza de la profesión. Sin embargo, el desarrollo de los estudios sobre periodismo es aún incipiente, lo que se evidencia en los escasos trabajos sistemáticos que permitan realizar un diagnóstico de la situación de la profesión en el país.

Los estudios basados en algún tipo de encuesta por muestreo se concentran en los últimos quince años y provienen en gran medida de iniciativas de la sociedad civil. En su mayoría fueron realizados por consultoras con participación de unas pocas universidades. Aunque de diverso enfoque y alcance, casi todas las investigaciones coinciden en remarcar la escasez no solo de referencias de la situación de los periodistas sino de datos para dimensionar adecuadamente el universo de la profesión. Por ello, la mayoría de las encuestas se centran principalmente en los periodistas de los grandes medios del distrito capital, más accesibles y mensurables, pero poco representativos del conjunto. Con estos antecedentes, las investigaciones disponibles se presentan como entrevistas exploratorias y se da a los resultados un carácter provisional. La falta de datos estadísticos de los profesionales en ejercicio podría atribuirse a la carencia de registros centralizados de la actividad por la baja participación de los periodistas en instituciones del ámbito sindical y de la sociedad civil. Pero también ha influido el hecho de que los marcos teóricos propios de la sociología de las redacciones y los estudios de la producción noticiosa recién empezaron a difundirse en el país en la última década. Esta debilidad de la base epistemológica ha diversificado las metodologías de abordaje al problema, que no siempre fueron las adecuadas para brindar un diagnóstico general de la profesión, aún pendiente.

Palabras clave: Periodismo; cultura periodística; metodología de la investigación; teorías de la comunicación.

\section{Argentine journalism: in search of data about the profession} Abstract

Argentina has a long journalistic tradition, with academic institutions that are pioneers in the profession. Nevertheless, the development of studies on 
journalism remains incipient, which is evinced by the few systematic studies that allow for a diagnosis of the profession's situation in the country.

Studies based on sample surveys concentrate on the last fifteen years and spring, in large part, from the efforts of civil society. In the majority of cases, they were conceived by consultants with the participation of a few universities. Although diverse in reach and focus, almost all of these investigations agree on the scarcity, not only of references to the journalists' situation, but also of data that can adequately measure the universe of the profession. Because of this, most surveys are principally centered on the journalists of mainstream media from the capital city, who are accessible and measurable but not representative of the entire field. With these antecedents, the available investigations are presented as exploratory interviews, while their results are given a provisional status.

The lack of statistical data on working professionals could be attributed to a dearth of centralized registries due to the low participation of journalists in labor unions and civil society. Also influential is the fact that the theoretical frameworks common to the sociology of editorial staffs and to studies of news production have only recently began to be disseminated across the country within the last decade. This weakness in the epistemological base has diversified the methodologies employed for broaching the problem, which have not always been adequate in providing a general diagnosis of the profession, which is still pending.

Keywords: journalism; journalistic culture; investigative methodology; theories of communication.

\section{Jornalismo argentino: na busca de dados sobre a profissão Resumo}

A Argentina tem uma forte tradição em jornalismo, com instituições acadêmicas pioneiras no ensino da profissão. Porém, o desenvolvimento dos estudos de jornalismo é ainda incipiente, com poucos trabalhos sistemáticos para fazer um diagnóstico da situação da profissão no país.

As pesquisas baseadas em mostras só aparecem nos últimos quinze anos e elas surgiram por iniciativa da sociedade civil e consultoras, com pouco interesse das universidades nesses estudos. Diversas em foco e matérias, quase todas as pesquisas oferecem poucos dados da situação dos jornalistas e menos ainda do conjunto dos profissionais. Muitas delas só pesquisam jornalistas da mídia da capital do país, mais acessíveis para o pesquisador, mas não representativos do conjunto (meios).

A falta de dados estadísticos do jornalismo argentino tem muitas razões: carência de censos da profissão e o pouco interesse dos jornalistas na participação em sindicatos e ONGs. No entanto, há também o fato de que só na última década começaram a serem distribuídas e aceitas as teorias e métodos para o estudo da sociologia das redações e a produção das notícias. Esta debilidade epistemológica retardou o desenvolvimento de metodologias para fazer um diagnóstico geral da profissão, ainda pendente.

Palavras-chave: jornalismo; cultura jornalística; métodos de investigação; teorias da comunicação. 


\section{Antecedentes}

Hacer un diagnóstico de la cultura periodística argentina de las últimas décadas es una tarea compleja. Los datos disponibles sobre la cantidad de periodistas en ejercicio y sus condiciones de trabajo son escasos y provienen, en su mayoría, de encuestas desarrolladas por fuera de los ámbitos académicos. En la investigación universitaria sobre el campo predominaron abordajes cualitativos y de análisis del discurso que no siempre permiten definir variables para dimensionar la profesión en su conjunto. Son escasos en el país los estudios sistemáticos sobre las condiciones profesionales de los periodistas, al punto que no existe una estimación de la cantidad de personas en ejercicio.

Este trabajo es parte de la investigación que corresponde al capítulo argentino de World of journalisms ${ }^{1}$, red global de académicos dedicados a los estudios del periodismo. La propuesta de este grupo es desarrollar una encuesta global sobre cultura periodística en base a un cuestionario común que permita realizar comparaciones de los datos obtenidos en las diferentes regiones y países participantes. Dado que se trata de una metodología cuantitativa que requiere de la determinación de una muestra sistemática, el equipo de investigación de la Argentina se enfrentó con la dificultad inicial de no contar con un censo de periodistas que ofreciera un universo poblacional con cuotas definidas. Esa circunstancia llevó a rastrear antecedentes de estudios similares para ver cómo se había calculado la muestra y en base a qué parámetros se había dimensionado el colectivo de los periodistas argentinos. En este artículo se presentan algunas de las conclusiones que arrojó ese relevamiento.

Antes de pasar a la investigación desarrollada, no puede dejar de mencionarse que la situación argentina de la investigación sobre el periodismo no es ajena a la del continente en general. Como señalan las investigadoras miembros de la red Worlds of journalism (Mellado, Moreira, Lagos \& Hernández, 2012), los estudios cuantitativos sobre cultura y actitudes del periodismo han sido esporádicos en Latinoamérica. Frank Priess fue responsable por la Fundación Konrad Adenuaer de la encuesta que se identificó como primera entre los escasos estudios similares. En esa publicación planteaba que "No es fácil encontrar en América Latina material de lectura que intente hacer un análisis sólido sobre una adecuada base representativa" (Fraga, 1997). El caso de Argentina no escapa a esta circunstancia, espe-

1 http://www.worldsofjournalism.org/ 
cialmente porque hace apenas una década la academia local dominante comenzó a considerar el marco teórico que corresponde a los estudios de la sociología de los emisores y a la producción noticiosa que suelen ser el marco conceptual que promueve este tipo de estudios.

En todo caso el periodismo no siempre fue abordado como problema de investigación que requiere de una perspectiva multidisciplinar y una metodología adecuada al objeto de estudio. La prioridad dada a la perspectiva crítica y a los estudios culturales orientó la investigación principal hacia el estudio de casos, el análisis del discurso o la discusión sobre la propiedad de los medios (Amado, 2011: 131). En este sentido observa el investigador César Arrueta que "En América Latina, particularmente Argentina, si bien a partir de 1980 y principios de la década del 90 se iniciaron indagaciones en este sentido, la atención estuvo concentrada prioritariamente en las consecuencias culturales de los mensajes" (Arrueta, 2010b: 220). Esta percepción se inscribe en la tendencia general, como confirma la observación de un informe sobre periodismo en toda Latinoamérica: "Lo notable es que hasta ahora prácticamente no se ha investigado el tema. Los medios se analizan tradicionalmente más bien por su significado social y los efectos logrados, es decir, los contenidos mediales, pero no con respecto de sus procesos de producción" (Instituto Prensa y Sociedad, 2004: 6).

Coincidentemente con la aparición del libro de Stella Martini, profesora de la Universidad de Buenos, con una síntesis apretada de los estudios más relevantes realizados de la teoría del news-making (Martini, 2000), con el cambio de siglo empezó a considerarse en los foros académicos argentinos el enfoque de los estudios de la noticia en su diversidad. Estas perspectivas contaban con más de cuatro décadas de publicaciones en las principales revistas científicas del campo, como surge de la sistematización que aparece en el libro de Mauro Wolf (1987). Sin embargo, son muy pocos los estudios disponibles que consideraron esos marcos conceptuales. La escasez de antecedentes locales fue señalada en la siguiente obra de Martini, que realizó junto con Luchessi, donde sostenían que en los últimos treinta años los estudios argentinos se ocuparon de la noticia y su análisis discursivo e ideológico a partir de investigaciones que recaban testimonios de periodistas, historias de medios en particular, relevamiento de opinión de los profesionales, entrevistas (literario-antropológicas, periodísticas) y ensayos sobre el periodismo y su dimensión política. Concluían entonces sus autoras que "La investigación sobre el periodismo en nuestro medio no ha alcanzado, con todo, un espacio de jerarquía relevante en 
el ámbito académico y tampoco en el de la práctica misma" (Martini \& Luchessi, 2004: 203).

A conclusión similar llega Arrueta a partir de un relevamiento de los encuentros científicos argentinos de dos décadas y también ubica hacia el fin del siglo pasado el momento en que se habilitó la discusión de las teorías relacionadas con el estudio de la noticia en los foros locales. Es elocuente la referencia de unas investigadoras que en el foro de la Red Nacional de Investigadores en Comunicación de 1997 hablaban de las "dificultades de abordaje científico que presentaba este fenómeno, considerado reciente e incipiente" (Arrueta, 2010a: p. 72). El investigador Arrueta ${ }^{2}$ analizó que los términos "reciente e incipiente" daban cuenta, más que de un proceso sistemático de estudios sobre noticias, de un interés colectivo en el campo periodístico-académico por indagar sobre cuestiones de producción de noticias, promovido por la visibilidad de alto impacto que la tarea informativa adquirió en los años noventa. Recuerda que en esos años el periodista fue epicentro de discusiones fuertemente mediatizadas que tuvieron una intensidad inédita en el país. Sin embargo, aclaró, ese interés "incipiente" por conocer las condiciones de producción de las noticias y las condiciones laborales de los periodistas "no significó luego un correlato de investigaciones con base empírica. Es más, es claramente notoria esa ausencia si uno compara la densidad de estudios norteamericanos y europeos con los producidos en Argentina y América Latina”. Esta constatación también sirve para ubicar el comienzo del interés académico sobre el periodismo argentino en la última década del siglo XX, aunque la discusión pública insista en ubicarlo en los años recientes.

Ante esta escasez de datos sobre las rutinas periodísticas, Arrueta destaca el aporte que han hecho algunas iniciativas cuantitativas de calidad periodística, que trabajan con análisis de contenido. En este sentido, el investigador considera que brindan parámetros cuantitativos que son "no solo de correlato entre rutinas y contenidos, sino también entre en el interior de los propios procesos productivos. Una primera mirada exploratoria en este sentido permite organizar luego la mirada cualitativa" (Arrueta, 2010b: 236). Sin embargo, este camino también ha sido poco transitado en las publicaciones académicas argentinas.

Arrueta califica esta tendencia en la investigación como descriptivo-anecdóti$\mathrm{ca}$, propia de una etapa exploratoria en términos epistemológicos, que en alguna

\footnotetext{
2 César Arrueta, profesor de la Universidad de Jujuy e investigador del CONICET, fue entrevistado para esta investigación en marzo de 2012.
} 
medida determinó que en los estudios de comunicación en la Argentina persistieran en la tendencia de indagar las derivaciones culturales de los contenidos periodísticos, más que en el estudio de la producción de esos contenidos ${ }^{3}$. De hecho, los observatorios de medios que se crearon a partir de 2006 en algunas universidades se concentran básicamente en el análisis del mensaje con metodologías centradas en las noticias y en los contenidos de los medios ${ }^{4}$. Este foco puesto en el mensaje antes que en su productor y en sus condiciones fácticas de trabajo que señala Arrueta explicaría la falta de datos empíricos para dimensionar el campo en lo que hace a cantidad de periodistas, su perfil, su cultura y demás variables concretas que estudian los abordajes de la producción noticiosa.

\section{Encuestas sobre la profesión periodística realizadas en Argentina}

A falta de un registro de los profesionales en ejercicio que permitiera determinar una muestra sistemática, como paso previo a la aplicación en la Argentina de la encuesta "Worlds of journalisms" se realizó una indagación de antecedentes locales de trabajos similares para ver cómo habían resuelto esa cuestión técnica ${ }^{5}$. Para ello, se rastrearon las principales encuestas realizadas sobre el ejercicio del periodismo a fin de ver sobre qué datos se había dimensionado el universo de los profesionales. El objetivo de esta revisión fue evaluar la población estudiada de modo de encontrar paradigmas que ayudaran a establecer una muestra más representativa para la futura encuesta. Por esa razón, en los estudios identificados solo se analizaron los aspectos metodológicos utilizados para recabar los datos y las ba-

\footnotetext{
3 Íbidem.

4 Según la definición que aparece en el sitio web de la Red de observatorios, "un observatorio de medios implica un estudio acerca del comportamiento de los medios de comunicación sobre un tema en particular en un determinado período de tiempo". Tampoco aparece el periodismo como objeto de estudio en la descripción de los proyectos que llevan a cabo cada una de las universidades participantes en la red, que enuncian que se ocupan del estudio de los mensajes y potenciales representaciones que se desprenderían de estos. Según describen los proyectos realizados hasta el momento, estos consisten en: análisis por el "modelo de "intencionalidad editorial" (Universidad Nacional de La Plata); "tratamiento periodístico" de ciertos temas (Universidad Nacional de Lomas de Zamora); el "papel que desempeña el proceso periodístico en la construcción de sentido común (sic) acerca de los derechos humanos en la Argentina" (Universidad Nacional de Cuyo); "debate y la reflexión teórica sobre la función social de los observatorios de medios" y "monitoreo de la información y de su resignificación en los medios de comunicación sobre temáticas de interés social y ciudadano" (Universidad Nacional de Córdoba); y "problemáticas de género, de tratamiento periodístico de pueblos originarios, experiencias de comunicación y desarrollo a la vez que se ocupa del análisis de las producciones audiovisuales sobre jóvenes" (Universidad Nacional de Salta). Las publicaciones que aparecen en el sitio no corresponden a la perspectiva de los estudios relevados [en http://www.redobservatorios.org.ar/web/?page_id=6, consultado el 25 de noviembre de 2012]

5 Los resultados preliminares fueron presentados en el primer Congreso internacional sobre estudios de periodismo "Identidad, cambio y desafíos de la profesión en el siglo XXI", Universidad de Santiago, Santiago de Chile, 27 al 29 de junio de 2012.
} 
ses tomadas para la determinación de las muestras, sin entrar en la consideración de los resultados específicos de cada estudio.

Del relevamiento de encuestas a periodistas realizadas en la Argentina se seleccionaron los trabajos que tomaban de manera directa la opinión de los sujetos sobre cuestiones específicas de su profesión a partir de algún tipo de encuesta por muestreo. Se descartaron los estudios de caso y los análisis discursivos de noticias a partir de los cuales se infieren condiciones de producción en la medida en que esos mismos estudios se plantean sus resultados como particulares y no representativos del conjunto.

Para consolidar el listado se revisaron las referencias bibliográficas de los principales trabajos publicados en el país en los últimos veinte años sobre periodismo local y se consultaron a informantes clave, especialmente a los que habían participado en los trabajos previos. Un eje temático de estos trabajos podría ser una definición de los antecedentes que da una de las investigaciones cuando dice que "la mayoría de los estudios anteriores busca encontrar respuestas que permitan definir el perfil del profesional de la información y las características de su trabajo diario" (Estudio de Comunicación \& Universidad Austral, 2008: 16).

Con estos criterios se identificaron once investigaciones de las cuales diez se ajustaban mayormente a los criterios de selección. Como se observa de la lista que sigue, la primera publicación de una investigación con alguna metodología cuantitativamente representativa aparece en 1996 y la última, en 2011. A los efectos de tener una idea de autores y temas, a continuación se enumeran por el año del trabajo de campo (en algunos casos, distinto de la fecha de publicación), sus responsables y el título del estudio. En un cuadro anexo se presentan algunos aspectos técnicos de las investigaciones, tales como institución responsable, los objetivos declarados de la investigación, el tipo de método de relevamiento de datos y el corpus de análisis. De ese cuadro pueden conocerse aspectos específicos de cada trabajo.

En este artículo se intentará caracterizar los alcances y métodos de las investigaciones, a las que se asignó un código de identificación con la sigla de la organización promotora y el año de realización a los efectos de agilizar la mención a lo largo del trabajo.

1. 1996: Rosendo Fraga. Centro de Estudios para la Nueva Mayoría, Fundación Konrad Adenauer, "Autopercepción del periodismo en Argentina". [FKA96] 
2. 1997: Gustavo Béliz y Enrique Zuleta Puceiro. Universidad Austral. "La cultura profesional del periodismo argentino: hacia un índice riesgo-país en materia de libertad de prensa”. [AUS97]

3. 1998: Luis Majul y Viviana Gorbato, Lila Luchessi, coordinadora general. Universidad de Belgrano. "Periodistas: qué piensan y qué hacen los que deciden en los medios". [UBE98]

4. 2000: F. Ruiz (coordinador), C. Álvarez Teijeiro, L. Elizalde, D. Fernández Pedemonte (Universidad Austral), H. Alconada (diario La Nación). Fundación Konrad Adenauer. "Prensa y congreso: trama de relaciones y representación social". [FKA00]

5. 2003: C. Schmidt-Liermann, M. Rovere, D. Lavalle Cobo, para Fundación Konrad Adenauer. "Periodismo y acceso a la información pública". [FKA03]

6. 2003: Instituto Prensa y Sociedad, Fundación Konrad Adenauer, "Cómo trabajan los periodistas latinoamericanos". [IPY03]

7. 2003: Stella Martini y Lila Luchessi, Universidad de Buenos Aires. "Los que hacen la noticia" [UBA03]

8. 2005: Giacobbe y Asociados para el Foro de Periodismo Argentino (FOPEA). "Sobre los periodistas y su profesión". [FOP05]

9. 2008: "Estudio de comunicación, Universidad Austral. "Periodistas y empresas: Claves de una relación necesaria índice". [AUS08]

10. 2011: CIO para FOPEA. "Encuesta sobre los periodistas y su profesión” [FOP11]

A estos trabajos podría agregarse la investigación de Graciela Paredes, de la Universidad del Salvador, que también se ocupa de "La imagen de los profesionales de la información en la prensa" [UDS09]. La autora realiza una categorización de temas a partir de testimonios de periodistas, pero no se incluye en el listado porque no trabaja con la metodología de encuesta, sino que utiliza las declaraciones de periodistas publicadas en noticias de diarios nacionales. Si bien no es equiparable metodológicamente al grupo seleccionado, se la consideró pertinente para ilustrar algunas cuestiones relacionadas con la determinación de perfiles profesionales.

La accesibilidad del material se estableció como un criterio adicional de selección en la medida en que es un dato elocuente del alcance del trabajo y su potencial difusión. Por ello, se incluyeron solo las publicaciones editoriales y científicas, en formato impreso o digital, previa verificación de estas referencias con investigadores y docentes vinculados con el tema. Por esta razón no se tuvieron en cuenta 
algunos informes elaborados por las asociaciones gremiales que realizaron algunas encuestas sobre aspectos parciales de la profesión, que no cumplían este requisito editorial.

De los títulos que aportaron los especialistas consultados no se consideraron aquellas publicaciones específicas por su circulación acotada ${ }^{6} \mathrm{y}$ las investigaciones desarrolladas con metodologías distintas de la encuesta de muestra. Se descartaron, por ejemplo, el caso de dos iniciativas de la Universidad Católica Argentina, de Buenos Aires, a cargo de la periodista y docente Raquel San Martín y de las investigadoras Lidia de la Torre y María Teresa Téramo, incluidas en una publicación sobre calidad periodística (Amado, 2007). La investigación de San Martín ${ }^{7}$ buscaba "evaluar la noción de información que manejan los periodistas naturalmente [...] a partir de grupos de discusión con periodistas de los diarios Clarín, La Nación y Página/12" (Amado, 2007: 142). El trabajo de Téramo y La Torre ${ }^{8}$ se ocupó de identificar parámetros de calidad en las noticias, algunos de los cuales permitían inferir prácticas periodísticas. La exhaustiva revisión de las investigaciones sobre periodismo en Argentina realizada por César Arrueta para su tesis doctoral (Arrueta, 2010a) aportó la referencia de algunos trabajos en medios de provincias ${ }^{9}$. En todos los casos se trataba de investigaciones cualitativas sobre gru-

\footnotetext{
6 La vicedecana de la Facultad de Periodismo de la Universidad Nacional de La Plata, Patricia Vialey, aportó las siguientes referencias de trabajos de investigación relacionados con la cultura periodística hasta 2011 junto con dos tesis de grado: "El sujeto profesional del derecho a la comunicación en la sociedad de la información: estudio del ejercicio de derechos de los graduados de la Facultad de Periodismo y Comunicación Social de la Universidad Nacional de la Plata". Directora: Eliades Analía (2011-2012). "Identificación de perfiles y tendencias en las articulaciones entre los campos profesional y académicos en la formación universitaria en comunicación social: un estudio de caso". Directora Nancy Díaz Larrañaga (2003-2004). Su responsable confirmó que fue basado en análisis documental y entrevistas.

7 San Martín, Raquel (2004). "La información como bien público: qué dicen los medios y qué piensan los periodistas", realizada en el marco del Programa de Estímulo a la Investigación y Aportes Pedagógicos del Instituto de Comunicación Social de la Universidad Católica Argentina (UCA). Informe.

8 De la Torre, Lidia \& Téramo, María Teresa (2004). La noticia en el espejo: medición de la calidad periodística: la información y su público. Buenos Aires: EDUCA.

De la Torre, Lidia (2007). Públicos y periodistas, semejanzas y diferencias: el Diario de Cuyo. Buenos Aires, EDUCA.

9 Arrueta, Julio (2009). "Rutinas de producción y calidad periodística en diarios de referencia dominante", Universidad Austral, tesis de doctorado en Comunicación.

Eisayaga, Amalia (2007). "Desafío en la adversidad: Notas sobre los medios gráficos en San Salvador de Jujuy" (p. 57-82). En: Arrueta, Cesar et al. (compiladores). Sobresentidos: estudios sobre comunicación, cultura y sociedad. San Salvador de Jujuy: EDIUNJu.

Postolski, Glen \& Rodríguez, Daniel (2005: 65-80). “Ambivalencias y potencialidades de las nuevas tecnologías en la organización del trabajo en la prensa argentina". En: Arrueta, Cesar (compilador). Sociedad, estado y medios: aportes para pensar la responsabilidad comunicativa en Jujuy. San Salvador de Jujuy: Ediciones de la Rueca.

Quiroga, Sergio (2000). "La producción de noticias en CTC Canal 2 (San Luis)". Citado por Arrueta (2009), ver cita ut supra.
} 
pos específicos cuyas conclusiones no se presentaban como diagnóstico del periodismo argentino en general.

\section{Alcance y métodos usados en las encuestas sobre periodismo argentino}

De las diez investigaciones seleccionadas solo la mitad podrían considerarse estrictamente encuestas representativas (FKA96, AUS97, FOP05, AUS08, FOP11). El resto considera una muestra demasiado pequeña de informantes argentinos o se presentan como indagaciones exploratorias o eminentemente cualitativas, con mayor o menor grado de sistematización en los cuestionarios. Solo tres han considerado los medios de todo el país (FOP05, AUS08, FOP11), aunque apenas las dos últimas han tomado una muestra aleatoria sistemática. Las restantes se centraron en los medios del distrito Capital, que si bien son los de mayor envergadura y tienen potencial de llegada a todo el país, no necesariamente son representativos de la cultura profesional del conjunto. De hecho, hay evidencias para presumir que las condiciones de trabajo de los grandes medios son diferentes de los de las provincias, donde a su vez son diferentes para los grandes medios locales que para los pequeños ${ }^{10}$, como muestra el Monitoreo de libertad de expresión que realiza FOPEA $^{11}$.

En algunas investigaciones, la muestra de Argentina correspondía a una pequeña parte de un estudio continental (FKA03, IPY03). En otros casos, se contrastó la percepción de los periodistas con la de otros grupos sociales (FKA96) o se analizó específicamente el vínculo informativo con otros grupos como el Congreso nacional (FKA00) o las áreas de prensa de las empresas (AUS08). Tampoco puede decirse que todos los estudios comparten variables para la definición de la cultura profesional argentina, dado que cada uno está planteado con objetivos específicos y se ocupan de diferentes aspectos.

Con respecto de la institución en las que se desarrollaron, dos se presentan como publicación de una facultad de comunicación (AUS97, AUS08); tres como trabajos realizados con el concurso de estudiantes de una cátedra (AUS97, UBE98,

\footnotetext{
10 Solo en el mercado de diarios, que es el que está más sistematizado, para comienzos de siglo se estimaban unos 274 títulos, de los cuales unos 150 correspondían a la provincia de Buenos Aires y 26, a la ciudad homónima. Asimismo, la Asociación de Diarios del Interior de la República Argentina (ADIRA) sostenía que el 80\% de sus asociados eran pequeñas y medianas empresas (Secretaría de Cultura de la Nación, 2003: 164).

11 Organización civil de periodistas, con aproximadamente trescientos asociados, que realiza un monitoreo de libertad de expresión en el país. Tomado de internet en septiembre de 2012: http://www.fopea.org/Libertad_de_ Expresion/Informes_Monitoreo_LE.
} 
UBA03); y cuatro fueron coordinados por profesores de alguna materia (UBE98, FKA00, UBA03, AUS08). Más allá del liderazgo de la Universidad Austral en este tipo de estudios, la iniciativa mayoritaria proviene de organizaciones de la sociedad civil (cuatro por la Fundación Konrad Adenauer y dos por FOPEA), mientras que en la realización hay una importante participación de consultoras privadas (FKA96; AUS97; FOP05; AUS08; FOP11).

En el conjunto, el trabajo de Paredes (UDS09) no responde a la metodología de encuesta sino que se inscribe dentro de los numerosos trabajos que utilizan el análisis de noticias para buscar las representaciones discursivas. Su detalle se incluyó en el anexo en tanto que utiliza la técnica de análisis cuantitativo de una muestra importante de noticias a partir de las cuales se identifican testimonios de periodistas que se ocupan específicamente de reflexionar sobre los alcances de la profesión. Algo similar realiza FKA00, aunque en este caso el análisis de noticias se usa como método de triangulación. Ambas justifican el método, como lo hace la mayoría de los estudios revisados, en que se tratan de indagaciones exploratorias de un tema poco estudiado.

De las investigaciones analizadas, las que consideran una muestra importante - basada en un criterio representativo más comprensible- son la primera de la serie (UBE96) y las últimas (AUS08, FOP11). En general, se ofrece una explicación de la metodología (FKA96, AUS97, FKA00, UBA03, AUS08, FOP11), aunque solo la mitad presenta una justificación técnica del criterio de muestreo (FKA96, UBE98, FKA00, AUS08, FOP11). Con una muestra también acotada, la investigación FKA00 es la que ofrece una justificación más técnica de la metodología elegida (Ruiz, F. et al., 2001: 14-18), en tanto que UBA03 es la que más problematiza la cuestión epistemológica, presentando las limitaciones que han tenido los estudios del periodismo en el país. Las autoras aclaran que el método fue la observación participante, aunque insisten en su carácter experimental, en la medida en que no lo consideran estrictamente etnográfico por aplicarse a un grupo social con “exceso' de voz pública” (Martini \& Luchessi, 2004: 184). Ambas investigaciones (FKA00, UBA03) utilizaron la técnica de la observación directa como método de triangulación y trabajaron sobre los medios de la ciudad de Buenos Aires. El diseño de la muestra de UBA03 considera criterios de audiencia para focalizarse en los "grandes medios con trayectoria y reconocimiento" (Martini \& Luchessi, 2004: 189). A partir de esta base seleccionaron "un grupo al que evaluamos como representativo de la profesión", aunque en el párrafo siguiente indican "que los in- 
formantes podrían haber sido otros" (loc. cit.). Las autoras son cautas a la hora de presentar el camino de investigación y aclaran que las conclusiones deben tomarse como parciales, solo para esos casos particulares (Martini \& Luchessi, 2004: 186) y los temas abordados parecen surgir de una clasificación elaborada por ellas a partir de los resultados. La investigación de Paredes (UDS09) también realiza una categorización de temas que surgen de las declaraciones de los periodistas, con lo cual en ambas podría suponerse que los temas surgieron de los informantes.

Los investigadores decidieron trabajar con los medios donde se realizó la investigación, justificando en que se trataba de los de mayor audiencia del país. La decisión metodológica de la mayoría de los trabajos determinó poner el foco en los periodistas de los grandes medios de la ciudad de Buenos Aires. En este sentido, la justificación de IPY03 puede tomarse como el parámetro usado para justificar el recorte en esos medios:

El número de encuestados elegidos (20) nos obliga a señalar que este resumen es apenas una muestra bastante sesgada y caótica de estos hábitos de trabajo en la ciudad de Buenos Aires, el lugar en la Argentina con mayor concentración de medios de comunicación y, por ende, mayor cantidad de trabajadores de prensa (Instituto Prensa y Sociedad, 2004: 16).

Solo UBA03 apoya el recorte "en razones científicas y focalización política en tanto este trabajo se basa en la relación de los periodistas con "el poder hegemónico" planteando que se haría luego un análisis de "la producción periodística alternativa" (que no se concretó a la fecha), para contar con parámetros de comparación (Martini \& Luchessi, 2004: 13). Lo cierto es que este universo tiene la ventaja de ser accesible (lo que se aclara en varias investigaciones) y ser más acotado en cantidad de medios. Sin embargo, tampoco en estos casos aparecen dimensiones concretas de los periodistas en ejercicio. En 1999, Lila Luchessi, coordinadora de la investigación UBE98 y luego de UBA03, planteaba esta dificultad: "Como en tantos otros campos, no existen relevamientos de la cantidad de profesionales en ejercicio en el país" (Majul \& Gorbato, 1999: 199). Una década después, todavía no se conoce alguna iniciativa que intente dimensionar el universo de los periodistas en ejercicio o de los espacios en que se desempeñan.

Cuando el Foro de Periodismo Argentino decidió emprender su primera investigación en 2005 armó un listado a partir de los periodistas asociados y de referencias que estos pudieran aportar en sus provincias. Andrés D’Alessandro, director 
de la organización ${ }^{12}$, señaló al respecto que partieron "de la base de que no sabemos con exactitud qué cantidad de periodistas en ejercicio hay en la Argentina, y mucho menos cuántos a nivel provincias". En función de ello, para la edición 2011 Cecilia Mosto, responsable de la consultora que llevó a cabo el relevamiento, propuso partir de los listados disponibles en FOPEA y sumar referencias de los socios de todo el país. A fin de subsanar falencias metodológicas de los estudios previos $^{13}$, su propuesta fue seleccionar los casos de manera sistemático aleatoria para garantizar representatividad y fijar un piso estadísticamente aceptable para poder analizar la información por zonas. De esa manera, conformaron una base de datos de dos mil quinientos contactos de medios diversos de todo el país, de los que obtuvieron un millar de respuestas de todas las provincias argentinas, lo que la convierte en la encuesta más amplia realizada. Sin embargo, aun en este caso, no hay forma de saber, por ejemplo, si las cuotas demográficas de los entrevistados coinciden con las del universo.

Del relevamiento realizado surge que entre las iniciativas identificadas hay un predominio de consultoras (FKA96, AUS97, FOP05, FKA08, FOP11) y de organizaciones de la sociedad civil (FKA96, FKA00, FKA03, IPY03, FOP05, FOP11) por sobre las casas de estudio. Esta conclusión coinciden con las conclusiones de un estudio previo del doctor Arrueta donde había planteado que "no es descabellado pensar, en consecuencia, que los estudios de newsmaking en nuestro país se hayan potenciado principalmente por un interés creciente de empresas y organizaciones civiles de conocer la lógica interna de los medios y establecer puntos de contacto con esa trama compleja de construcción de realidad" (Arrueta, 2006). La articulación de estos otros actores con la universidad fue planteada por Damián Fernández Pedemonte - quien participó en dos de los estudios identificados- y permite comprender un poco mejor las carencias de los antecedentes:

Como una veta, el estudio sólo contiene elementos de valor. No es un "mamotreto" teórico o ensayístico. Es el resultado de un trabajo arduo de respeto a lo que dicen los informantes: de definir una muestra representativa, confeccionar cuestionarios claros y pertinentes, lograr que se completen, procesar las respuestas, analizar e interpretar los datos. La investigación aplicada es una función típica de la universidad, que dispone del tiempo, de la independencia y de la metodología para servir así a la sociedad. Y es, además, un punto de intersección con las con-

12 Andrés D’Alessandro, director de Fopea, fue consultado para este trabajo en febrero de 2012.

13 Cecilia Mosto, directora de la Consultora CIO, fue consultada para este trabajo en marzo de 2012. 
sultoras - como se ve en este estudio conjunto de Estudio de Comunicación y la Facultad de Comunicación de la Universidad Austral一: porque una investigación universitaria de estas características provee de la información necesaria para tomar decisiones, para la implementación de planes, que ya es tarea propia de las consultoras (Estudio de Comunicación \& Universidad Austral, 2008: 8).

De esta manera, por oposición, se evidencian ciertas debilidades de los estudios previos del campo, a las que hay que sumar la pobre proyección de la producción científica local en los estudios globales de periodismo. En este contexto no resulta tan extraño que solo tres de las encuestas aporten conclusiones que podrían tomarse como representativas del sector y ninguna ofrezca certezas acerca de las dimensiones del universo en estudio. La producción en campo priorizó la observación al periodismo a partir de los contenidos que publica por sobre la indagación las condiciones en que son producidos. Cuando lo ha hecho, ha preferido metodologías de tipo etnográficas, de observación de casos, que no posibilitan obtener conclusiones del conjunto más que aproximativamente.

\section{Conclusiones para una agenda de investigación}

Sin certeza de la cantidad de profesionales ni las dimensiones del sistema de medios en los que se desempeñan, es difícil establecer bases técnicas para estudios que arrojen conclusiones representativas de las problemáticas del sector. Sin embargo, esta limitación no parece haber sido un impedimento para el desarrollo del campo en Argentina. Los diez trabajos relevados coinciden en señalar como características de los estudios sobre periodismo desinterés por las metodologías cuantitativas, escaso desarrollo de los estudios dentro de la teoría del newsmaking, falta de datos empíricos sobre el sector y dificultades para encontrar conclusiones que puedan ser tomadas como representativas del periodismo argentino.

Si hubiera que plantear algunas hipótesis acerca de la particular trayectoria que tuvieron los estudios del periodismo en el país, no puede obviarse la impronta teórica dominante en los centros académicos locales. Silvio Waisbord recuerda que la particular formación y consolidación de un campo interdisciplinario e internacional del estudio de la comunicación fue marcado por "el dominio de debates teóricos anclados en las academias estadounidenses y británicas, las cuales a su vez reflejaban preguntas empíricas propias de esos países" (Waisbord, 2012). En Argentina, la primera línea de investigación fue prácticamente excluida de la discusión (en muchos casos, también del currículo universitario) y no por razones 
estrictamente epistemológicas. Roberto Follari señaló al respecto que la preeminencia de las disciplinas en los distintos momentos históricos depende de "relaciones de fuerza que no son principalmente intrateóricas":

Eso lleva a que algunas de estas disciplinas alcancen preeminencia. Si nosotros, grosso modo, hacemos ahora una periodización en los estudios sobre comunicación, en Argentina allá por los años 70, hacia los comienzos de las carreras de comunicación en el país, tuvo mucho peso la crítica ideológica planteada desde el análisis sociopolítico en términos de Mattelart con Para leer el Pato Donald, o en términos de Pasquali (Arrueta, Brunet, \& Guzmán, 2010: 22).

El profesor Follari agrega que esas "condiciones políticas y culturales intervienen siempre sobre cualquier disciplina, pero en cuanto la condición epistemológica es más débil, intervienen más. Así, la posición conceptual queda subordinada a las condiciones políticas y culturales cuando hay escaso desarrollo de la propuesta epistemológica" (Arrueta, Brunet \& Guzmán, 2010: 23). Lo que ocurre es que la discusión teórica se vuelve esencial cuando de ella depende no solo el abordaje de un problema de estudio sino cuando configura un obstáculo para su definición. En este sentido, se vuelve urgente contar con datos locales dada la dificultad de hacer propias las conclusiones obtenidas para otros contextos, como bien observa Silvio Waisbord:

Si bien en un mundo globalizado el periodismo está conectado a través de múltiples redes profesionales e informativas, ni las normas éticas que rigen el trabajo periodístico cotidiano ni las aspiraciones individuales y colectivas del periodismo son idénticas (Donsbach y Patterson, 2004; Hanitzsch, 2007). La racionalidad de los procesos de decisión en redacciones es eminentemente local, influenciada por la arquitectura del sistema político, factores económicos, expectativas de las audiencias y otros factores (Waisbord, 2012).

Estas variaciones entre contextos han sido planteadas en el trabajo de Tomás Hanitzsch y Claudia Mellado, que señalan que los condicionamientos percibidos varían de un país a otro, aunque en todos los casos se mantenga una brecha entre lo percibido y la autonomía real del periodista (Hanitzsch \& Mellado, 2011: 17). Esta observación plantea la necesidad de ir más allá de la opinión de los profesionales o de un análisis de medios acotados a casos particulares. Las entrevistas que apoyan los numerosos estudios de casos y muchas de las tesis no constituyen una metodología que ofrezca conclusiones extensibles a otros contextos más allá de los analizados, como plantean las profesoras Martini y Luchessi (2004). 
Otros problemas de investigación surgen cuando se intenta comparar la situación del periodismo argentino con las tendencias globales. Por caso, en los últimos años se plantea la existencia de un "periodismo militante" que se autodefine como el que se opone al "periodismo de los medios hegemónicos", denominación general con que se alude a las grandes empresas mediáticas. Sin embargo, el determinismo entre la propiedad de los medios y el ejercicio de un periodismo particular sería una excepción a la tendencia global que marcan Hanitzsch y Mellado cuando plantean que las influencias económicas y de los dueños de los medios son en general poco percibidas por los periodistas en todos los países relevados. Al carecer de evidencias empíricas locales acerca de que existen dos formas contrapuestas de ejercer la profesión y qué características específicas tienen, la discusión queda en un intercambio de opiniones. Estudios previos les permiten concluir a Hallin y Mancini que la oposición profesional o periodismo instrumentalizado (política o comercialmente) no representan una oposición que se da en todas las circunstancias de la misma manera. La profesionalización y la orientación a fines no son dimensiones excluyentes en la medida que en que pueden aplicarse ciertos parámetros profesionales que mantienen la distinción profesional en términos bourdianos (Hallin \& Mancini, 2004: 37). Sin embargo, estos matices no siempre están presentes en la discusión sobre la profesión que se observa en los medios y en los foros en los últimos años.

No se trata en este caso de traer a cuento en este trabajo la vieja discusión de la validez de las metodologías cuantitativas por sobre las cualitativas, sino de señalar que el desinterés por las primeras en el campo del periodismo ha determinado que aún hoy en Argentina no se cuente con una dimensión del sector, ni se haya problematizado su falta. La debilidad de la representación gremial, la falta de consejos profesionales y la baja presencia de organizaciones profesionales pueden ser, en parte, razones de esta falta de datos en la medida en que estas instituciones suelen ser las bases que toman los estudios de los otros países para desarrollar sus muestras. La fuerte presencia de centros de formación de periodistas, con casi un centenar de instituciones en todo el país no necesariamente se refleja en una heterogeneidad de abordajes y metodologías de estudio ${ }^{14}$. Del relevamiento realizado surge que las encuestas sobre el ejercicio profesional provienen, en su mayoría, de

14 Existe casi un centenar de instituciones de enseñanza de periodismo. Luchessi y Martini consignan que para 2003 había 36 universidades nacionales, 39 universidades privadas, 23 institutos y 7 posgrados (Martini \& Luchessi, 2004: 32). 
fuentes no académicas. Son, además, muy pocas las encuestas que consideran los medios de todo el país, lo cual nos enfrenta a la limitación de carecer de un diagnóstico de la situación del periodismo argentino en su conjunto.

Esto acarrea una seria limitación metodológica a la hora de diseñar una encuesta dado que se carece de datos básicos tales como la proporción de periodistas que trabajan en medios grandes o pequeños, urbanos o del interior; cuál es la composición etaria y social de las redacciones o cómo está conformada la pirámide de categorías laborales. La insuficiencia de estos datos implica también que no se puede hablar de un perfil del ejercicio del periodismo en Argentina sino de diagnósticos parciales, siempre provisorios.

El hecho de que hayan sido organizaciones civiles y universidades privadas las que más se ocuparon de recabar algunos datos no está en el valor de sus trabajos sino en que sus resultados no suelen ser discutidos en los foros académicos tradicionales. Como correlato, no se ha problematizado aún la necesidad de conocer qué cantidad de profesionales hay en ejercicio, dónde están y cómo desarrollan su tarea. Estos estudios serían valiosas contribuciones para triangular los múltiples estudios sobre los contenidos mediáticos, en la medida que permitiría contrastar las inferencias acerca de las condiciones de producción que se observan en el discurso con los aportes de la sociología de las redacciones y la teoría de la noticia. En este contexto, la perspectiva académica podría tomar los escasos antecedentes de estudios de la sociedad civil para enriquecerlos teóricamente y proyectar algunas conclusiones de los estudios de periodismo argentino en la agenda mundial de investigación.

\section{Referencias}

Amado, A. (2007). Periodismo de calidad: debates y desafíos. Buenos Aires: La Crujía.

Amado, A. (2011). "Los compromisos del periodista y del investigador académico" (p. 119-128). En: Christofoletti, R. \& Karam, F. J. (eds.). Jornalismo investigativo e pesquisa científica: fronteiras. Florianópolis: Editora da Universidade Federal de Santa Catarina; Editora Insular.

Arrueta, C. (2006). "Estudios sobre emisores y procesos productivos en medios de comunicación: estado de la cuestión en Argentina”. En: X Jornadas Nacionales de Investigadores en Comunicación. San Juan: Red Nacional de Investigadores en Comunicación. Consultado el 9 de septiembre de 2012 en: http://www.redcomunicacion.org/memorias/pdf/2006ararrueta1.pdf

Arrueta, C. (2010a). ¿Qué realidad construyen los diarios? Buenos Aires: La Crujía.

Arrueta, C. (2010b). "Entender los medios, estudiar las noticias: una propuesta epistemológica y metodológica para el estudio de procesos de producción informativa” (p. 215-241). En: Arrueta, C., Brunet, M. \& Guzmán, J. (eds.). La comunicación como objeto de estudio. San Salvador de Jujuy: Dass. 
Arrueta, C., Brunet, M. \& Guzmán, J. (2010). La comunicación como objeto de estudio. San Salvador de Jujuy: Dass.

Beliz, G. \& Zuleta Puceiro, E. (1998). La cultura profesional del periodismo argentino: hacia un índice riesgo-país en materia de libertad de prensa. Buenos Aires: Facultad de Ciencias de la Comunicación, Universidad Austral. (Cuadernos Australes de Comunicación; 1).

Donsbach, W. \& Patterson, T. E. (2004). "Political news journalists: partisanship, professionalism, and political roles in five countries". En: Esser, F. \& Pfetsch, B. (comps.). Comparing political communication: theories, cases, and challenges. New York: Cambridge University Press.

Estudio de Comunicación \& Universidad Austral (2008). Periodistas y empresas: claves de una relación necesaria. Buenos Aires: Universidad Austral. Facultad de Comunicación; Estudio de Comunicación.

Foro de Periodismo Argentino (FOPEA) \& CIO Argentina (2011). Encuesta sobre los periodistas y su profesión. Buenos Aires: FOPEA. Consultado el 9 de septiembre de 2012 en: http://www.fopea. org/Etica/Encuesta_sobre_Periodismo

Foro de Periodismo Argentino (FOPEA) \& Giacobbe y Asoc. (2005). Sobre los periodistas y su profesión. Buenos Aires: FOPEA. Consultado el 10 de septiembre de 2012 en: http://www.fopea. org/content/download/331/2036/file/Informe.pdf

Fraga, R. (1997). Autopercepción del periodismo en Argentina. Buenos Aires: Editorial de Belgrano.

Hallin, D. \& Mancini, P. (2004). Comparing media systems: three models of media and politics. Cambridge: Cambridge University Press.

Hanitzsch, T. (2007). Deconstructing journalism culture: toward a universal theory. Communication Theory, 17 (4): 367-385.

Hanitzsch, T. \& Mellado, C. (2011). What shapes the news around the world? How journalists in eighteen countries perceive influences on their work. The International Journal of Press/Politics, 20 (10): $1-23$.

Instituto Prensa y Sociedad (2004). Cómo trabajan los periodistas latinoamericanos. Lima: Instituto Prensa y Sociedad.

Majul, L. \& Gorbato, V. (1999). Periodistas: qué piensan y qué hacen los que deciden en los medios. Buenos Aires: Sudamericana.

Martini, S. (2000). Periodismo, noticia y noticiabilidad. Buenos Aires: Norma.

Martini, S. \& Luchessi, L. (2004). Los que hacen la noticia. Buenos Aires: Biblos.

Mellado, C., Moreira, S., Lagos, C. \& Hernández, M. E. (2012). Comparing journalism cultures in Latin America: the case of Chile, Brazil and Mexico. International Communication Gazette, 74: 60-77.

Paredes, G. [2010]. La imagen de los profesionales de la información en la prensa. Buenos Aires: Universidad del Salvador. Facultad de Ciencias de la Educación y de la Comunicación Social. Consultado el 29 de febrero de 2012 en: http://fcecs.usal.edu.ar/sites/default/files/Archivos/fcecs/ otros/2.pdf

Ruiz, F., Alvarez Teijeiro, C., Elizalde, L., Fernández Pedemonte, D. \& Alconada, H. (2001). Prensa y congreso: trama de relaciones y representación social. Buenos Aires: La Crujía.

Schmidt-Liermann, C., Rovere, M. \& Lavalle Cobo, D. (2003). Periodismo y acceso a la información pública. Lima: Fundación Konrad Adenauer.

Secretaría de Cultura de la Nación (2003). Industrias culturales: mercado y políticas públicas en Argentina. G. Mastrini \& S. Contreras (eds.). Buenos Aires: CICCUS. 
Waisbord, S. (2012, ene.-mar.). Periodismo y política: repensar la agenda de investigación en la academia globalizada. Revista Telos. Consultado el 10 de septiembre de 2012 en: http:// sociedadinformacion.fundacion.telefonica.com/url-direct/pdf-generator?tipoContenido=articul oTelos\&idContenido $=2012013016170001$ \&idioma $=$ es.

Wolf, M. (1987). La investigación de la comunicación de masas. Barcelona: Paidós.

\section{Correspondencia}

\section{Adriana Amado Suárez}

Universidad de La Matanza

amadoa@catedraa.com.ar

\section{Anexo}

\section{Síntesis de las investigaciones sobre periodistas realizadas en Argentina (1996-2011)}

La presentación de los estudios relevados incluida en esta tabla anexa se realiza consignando el año de realización del estudio y el título que le dieron sus autores, que se consignan junto con la institución que auspició la investigación (entre paréntesis se incluye la referencia bibliográfica). Para una somera descripción de la metodología que utilizaron cada uno de los estudios se mencionan los objetivos declarados, la metodología utilizada en cada estudio y la determinación del corpus de análisis de acuerdo con lo que mencionan los propios autores. Excede el objetivo de este artículo el análisis de las conclusiones de cada uno de los estudios, sino que se consideraron los datos que usaron para dimensionar el colectivo de los periodistas de Argentina. 
Año de realización: 1996

\begin{tabular}{|c|c|c|c|}
\hline \multicolumn{4}{|c|}{ Título del estudio: "Autopercepción del periodismo en Argentina" [FKA96] } \\
\hline $\begin{array}{l}\text { RESPONSABLES / INSTITUCIÓN } \\
\text { AUSPICIANTE (REFERENCIA } \\
\text { BIBLIOGRÁFICA) }\end{array}$ & $\begin{array}{l}\text { OBJETIVOS DECLARADOS EN } \\
\text { CADA INVESTIGACIÓN }\end{array}$ & $\begin{array}{l}\text { METODOLOGÍA UTILIZADA POR } \\
\text { LOS ESTUDIOS RELEVADOS }\end{array}$ & $\begin{array}{l}\text { CORPUS DE ANÁLISIS } \\
\text { DE CADA ESTUDIO }\end{array}$ \\
\hline $\begin{array}{l}\text { Rosendo Fraga (comp.), responsable } \\
\text { por la consultora Centro de Estudios } \\
\text { para la Nueva Mayoría, con el apoyo de } \\
\text { la Fundación Konrad Adenauer. } \\
\text { Se consigna que en la edición del libro } \\
\text { participó la Fundación Editorial de la } \\
\text { Universidad de Belgrano. } \\
\text { (Fraga, 1997) }\end{array}$ & $\begin{array}{l}\text { Estudio de opinión para poner en } \\
\text { conjunción Autopercepción del perio- } \\
\text { dismo y comparación con percepción } \\
\text { de "líderes de opinión" (empresarios, } \\
\text { políticos y jueces) y opinión pública. } \\
\text { El trabajo se presenta como "des- } \\
\text { criptivo y exploratorio" (p. 45). En el } \\
\text { posfacio, la Fundación agrega que el } \\
\text { objetivo es "p oner de manifiesto las } \\
\text { debilidades que se evidencian en un } \\
\text { segmento de difíill relacionamiento } \\
\text { entre el periodismo y la política, con } \\
\text { el propósito de proveer posibles enfo- } \\
\text { ques de solución a alentar procesos de } \\
\text { reforma ya en marcha" (p. 195). }\end{array}$ & $\begin{array}{l}\text { Estudio exploratorio descriptivo, con } \\
\text { muestras representativas (así mencio- } \\
\text { nado por los autores). Relevamiento } \\
\text { por encuesta personal con un cues- } \\
\text { tionario de } 49 \text { preguntas que surgió de } \\
\text { una prueba piloto de un cuestionario } \\
\text { de } 60 \text { ítems. }\end{array}$ & $\begin{array}{l}\text { Periodistas: muestra de } 120 \text { periodistas } \\
\text { con cuotas de edad y medio sobre uni- } \\
\text { verso de periodistas de Buenos Aires } \\
\text { (ámbito metropolitano). Se trabajaron } \\
\text { con datos secundarios para estratifi- } \\
\text { car los periodistas por modalidad de } \\
\text { trabajo. } \\
\text { Líderes de opinión: } 147 \text { entrevistas, } \\
\text { sobre listado de la consultora, con la } \\
\text { condición de que "hubieran tenido } \\
\text { contacto con la prensa" (p. 48) } \\
\text { Opinión pública: } 503 \text { entrevistas. } \\
\text { Muestra probabilística estratificada por } \\
\text { edad, sexo, nivel educativo y situación } \\
\text { socioeconómica. } \\
\text { Junio-agosto de } 1996 \text {. }\end{array}$ \\
\hline \multicolumn{4}{|l|}{ Año de realización: 1997} \\
\hline \multicolumn{4}{|c|}{ "La cultura profesional del periodismo argentino: hacia un índice riesgo-país en materia de libertad de prensa" [AUS97] } \\
\hline $\begin{array}{l}\text { RESPONSABLES / INSTITUCIÓN } \\
\text { AUSPICIANTE (REFERENCIA } \\
\text { BIBLIOGRÁFICA) }\end{array}$ & $\begin{array}{l}\text { OBJETIVOS DECLARADOS EN } \\
\text { CADA INVESTIGACIÓN }\end{array}$ & $\begin{array}{l}\text { METODOLOGÍA UTILIZADA POR } \\
\text { LOS ESTUDIOS RELEVADOS }\end{array}$ & $\begin{array}{l}\text { CORPUS DE ANÁLISIS } \\
\text { DE CADA ESTUDIO }\end{array}$ \\
\hline $\begin{array}{l}\text { Gustavo Béliz y Enrique Zuleta Puceiro } \\
\text { por la consultora Sofres-lbope, con } \\
\text { alumnos de la cátedra "Análisis de in- } \\
\text { formación de la política" de la Facultad } \\
\text { de Ciencias de la información de la } \\
\text { Universidad Austral. } \\
\text { (Beliz \& Zuleta Puceiro, 1998) }\end{array}$ & $\begin{array}{l}\text { Caracterizar la cultura profesional } \\
\text { del periodismo argentino. Se plantea } \\
\text { como "el primer diagnóstico descripti- } \\
\text { vo de la cultura profesional del perio- } \\
\text { dismo argentino" (p. } 7 \text { ). } \\
\text { Se consideraron las siguientes va- } \\
\text { riables de medición: a) criterios de } \\
\text { noticiabilidad; b) niveles de influencia } \\
\text { y credibilidad del periodismo; c) condi- } \\
\text { ciones de competencia; d) percepción } \\
\text { de otras instituciones públicas; e) uso } \\
\text { de fuentes y recursos; e) vulnerabilidad } \\
\text { frente a episodios de corrupción; f) } \\
\text { condiciones laborales; g) el periodista } \\
\text { como persona; } h \text { ) autocrítica del des- } \\
\text { empeño profesional. }\end{array}$ & $\begin{array}{l}\text { El enfoque metodológico se presen- } \\
\text { ta como "cuantitativo-cualitativo". } \\
\text { Reconocen que las limitaciones de } \\
\text { proyección de la muestra y el método } \\
\text { (muchos de los entrevistados comple- } \\
\text { taron el cuestionario por su cuenta, sin } \\
\text { un entrevistador), hacen del trabajo } \\
\text { una"técnica mixta". } \\
\text { Se trató de entrevistas a través de la } \\
\text { aplicación de un cuestionario estruc- } \\
\text { turado, sobre una muestra represen- } \\
\text { tativa. } \\
\text { Se plantea el trabajo que propone un } \\
\text { autoanálisis que sirva para desarrollar } \\
\text { un índice de la libertad de prensa. }\end{array}$ & $\begin{array}{l}\text { El cuestionario se desarrolló a partir de } \\
\text { entrevistas a informantes clave. } \\
\text { Se realizaron } 131 \text { encuestas a perio- } \\
\text { distas de medios nacionales de Capital } \\
\text { Federal y Gran Buenos Aires. Se tuvie- } \\
\text { ron en cuenta la segmentación por tipo } \\
\text { de medio, edad, antigüedad, jerarquía } \\
\text { y niveles remunerativos. } \\
\text { Octubre y diciembre de } 1997 .\end{array}$ \\
\hline
\end{tabular}


Año de realización: 1997

\begin{tabular}{|c|c|c|c|}
\hline \multicolumn{4}{|c|}{ "Periodistas: qué piensan y qué hacen los que deciden en los medios" [UBE98] } \\
\hline $\begin{array}{l}\text { RESPONSABLES / INSTITUCIÓN } \\
\text { AUSPICIANTE (REFERENCIA } \\
\text { BIBLIOGRÁFICA) }\end{array}$ & $\begin{array}{l}\text { OBJETIVOS DECLARADOS EN } \\
\text { CADA INVESTIGACIÓN }\end{array}$ & $\begin{array}{l}\text { METODOLOGÍA UTILIZADA POR } \\
\text { LOS ESTUDIOS RELEVADOS }\end{array}$ & $\begin{array}{l}\text { CORPUS DE ANÁLISIS } \\
\text { DE CADA ESTUDIO }\end{array}$ \\
\hline $\begin{array}{l}\text { Luis Majul y Viviana Gorbato. Lila } \\
\text { Luchessi, coordinadora general. Las } \\
\text { entrevistas se hicieron con alumnos de } \\
\text { la materia Habilitación profesional I, de } \\
\text { la carrera de Periodismo de la Universi- } \\
\text { dad de Belgrano. } \\
\text { (Majul \& Gorbato, 1999) }\end{array}$ & $\begin{array}{l}\text { Indagar en los periodistas que toman } \\
\text { decisiones en los medios de mayor cir- } \\
\text { culación las siguientes variables: } \\
\text { - Poder de la prensa. } \\
\text { - Comportamientos corporativos. } \\
\text { - Ética implíitita en el ejercicio perio- } \\
\text { dístico. }\end{array}$ & $\begin{array}{l}\text { Entrevistas (cuestionario con } 75 \text { pre- } \\
\text { guntas) a } 115 \text { periodistas. } \\
\text { Entrevistas abiertas en profundidad a } \\
13 \text { profesionales. }\end{array}$ & $\begin{array}{l}115 \text { respuestas de } 248 \text { contactados del } \\
\text { universo de los "periodistas que deci- } \\
\text { den con alto nivel de responsabilidad } \\
\text { la noticia de mañana" (p. 199). Los } \\
\text { medios fueron diarios (4); revistas (3); } \\
\text { radio AM (4); cadenas (4); agencias de } \\
\text { noticias (4).Se eligieron con criterio de } \\
\text { representación de cada medio y activi- } \\
\text { dad dentro de cada empresa. }\end{array}$ \\
\hline \multicolumn{4}{|l|}{ Año de realización: 2000} \\
\hline \multicolumn{4}{|c|}{ "Prensa y congreso: trama de relaciones y representación social" [FKA00] } \\
\hline $\begin{array}{l}\text { RESPONSABLES / INSTITUCIÓN } \\
\text { AUSPICIANTE (REFERENCIA } \\
\text { BIBLIOGRÁFICA) }\end{array}$ & $\begin{array}{l}\text { OBJETIVOS DECLARADOS EN } \\
\text { CADA INVESTIGACIÓN }\end{array}$ & $\begin{array}{l}\text { METODOLOGÍA UTILIZADA POR } \\
\text { LOS ESTUDIOS RELEVADOS }\end{array}$ & $\begin{array}{l}\text { CORPUS DE ANÁLISIS } \\
\text { DE CADA ESTUDIO }\end{array}$ \\
\hline $\begin{array}{l}\text { Ruiz, F. (coordinador), Álvarez Teijeiro, } \\
\text { C., Elizalde, L., Fernández Pedemonte, } \\
\text { D. (profesores de la Facultad de comu- } \\
\text { nicación de la Universidad Austral), } \\
\text { Alconada, H. (diario La Nación). Apoyo } \\
\text { de la Fundación Konrad Adenauer. } \\
\text { (Ruiz et al., 2001) }\end{array}$ & $\begin{array}{l}\text { Indagar sobre la relación de la prensa } \\
\text { con el Congreso nacional, identifican- } \\
\text { do factores de poder y funciones de los } \\
\text { periodistas y los medios. }\end{array}$ & $\begin{array}{l}\text { Se detallaron varias etapas: } \\
\text { - Entrevistas en profundidad. } \\
\text { - Taller con } 17 \text { protagonistas de la acti- } \\
\text { vidad parlamentaria. } \\
\text { - Entrevistas con cuestionario autoad- } \\
\text { ministrado (66 preguntas). } \\
\text { - Análisis de noticias. } \\
\text { - Observación directa. }\end{array}$ & $\begin{array}{l}\text { Muestra no probabilística. } 93 \text { entre- } \\
\text { vistados ( } 33 \text { legisladores, } 40 \text { jefes de } \\
\text { prensa y } 20 \text { periodistas), del ámbito } \\
\text { del Congreso Nacional que funciona en } \\
\text { la ciudad de Buenos Aires. }\end{array}$ \\
\hline \multicolumn{4}{|l|}{ Año de realización: 2003} \\
\hline \multicolumn{4}{|c|}{ "Periodismo y acceso a la información pública" [FKA03] } \\
\hline $\begin{array}{l}\text { RESPONSABLES / INSTITUCIÓN } \\
\text { AUSPICIANTE (REFERENCIA } \\
\text { BIBLIOGRÁFICA) } \\
\end{array}$ & $\begin{array}{l}\text { OBJETIVOS DECLARADOS EN } \\
\text { CADA INVESTIGACIÓN }\end{array}$ & $\begin{array}{l}\text { METODOLOGÍA UTILIZADA POR } \\
\text { LOS ESTUDIOS RELEVADOS }\end{array}$ & \begin{tabular}{|l} 
CORPUS DE ANÁLISIS \\
DE CADA ESTUDIO
\end{tabular} \\
\hline $\begin{array}{l}\text { Cornelia Schmidt-Liermann, Marta } \\
\text { Rovere y Dolores Lavalle Cobo, para la } \\
\text { Fundación Konrad Adenauer. } \\
\text { (Schmidt-Liermann, Rovere, \& Lavalle } \\
\text { Cobo, 2003) }\end{array}$ & $\begin{array}{l}\text { El trabajo tuvo como foco el derecho de } \\
\text { acceso a la información y el uso que los } \\
\text { periodistas hacen del recurso legal. El } \\
\text { cuestionario tenía algunas preguntas } \\
\text { acerca del tipo de periodismo que } \\
\text { ejercían los informantes y cuestiones } \\
\text { relacionadas con el ejercicio profesio- } \\
\text { nal como el uso de fuentes, amenazas } \\
\text { y presiones, entre otros. }\end{array}$ & $\begin{array}{l}\text { Investigación cualitativa, por medio de } \\
\text { entrevistas personales y un sondeo no } \\
\text { probabilístico a periodistas, editores, } \\
\text { directivos y empresarios y profesio- } \\
\text { nales de la comunicación. Se realizó } \\
\text { mediante un cuestionario de doce pre- } \\
\text { guntas abiertas a partir de las cuales se } \\
\text { elaboraron conclusiones, apoyadas en } \\
\text { información de contexto específica del } \\
\text { tema de acceso a la información. }\end{array}$ & $\begin{array}{l}169 \text { periodistas, sin cuotas por medios } \\
\text { ni por función. En Argentina se logra- } \\
\text { ron } 104 \text { respuestas (47 entrevistas per- } \\
\text { sonales y } 57 \text { cuestionarios); México: } 30 \\
\text { respuestas; Uruguay, 25; Perú, } 10 . \\
\text { Duración: } 6 \text { meses. }\end{array}$ \\
\hline
\end{tabular}


Año de realización: 2003

"Cómo trabajan los periodistas latinoamericanos" [IPY03]

\begin{tabular}{|l|l|}
\hline $\begin{array}{l}\text { RESPONSABLES/INSTITUCIÓN } \\
\text { AUSPICIANTE (REFERENCIA } \\
\text { BIBLIOGRÁFICA) }\end{array}$ & $\begin{array}{l}\text { OBJETIVOS DECLARADOS EN } \\
\text { CADA INVESTIGACIÓN }\end{array}$ \\
\hline $\begin{array}{l}\text { Instituto Prensa y Sociedad, Fundación } \\
\text { Konrad Adenauer. } \\
\text { (Instituto Prensa y Sociedad, 2004) }\end{array}$ & $\begin{array}{l}\text { El trabajo se presenta con una pregun- } \\
\text { ta general: "¿Y qué tan exigentes son } \\
\text { los procesos de trabajo de los perio- } \\
\text { distas en América Latina y qué margen } \\
\text { existe para nuevos modelos de nego- } \\
\text { cios y mayor calidad?" Las categorías } \\
\text { de las variables son: aspectos técnicos } \\
\text { del área donde trabaja; hábitos de tra- } \\
\text { bajo; cualidades profesionales. }\end{array}$ \\
\hline
\end{tabular}

Año de realización: 2003

"Los que hacen la noticia" [UBA03]

\begin{tabular}{|c|c|c|c|}
\hline $\begin{array}{l}\text { RESPONSABLES / INSTITUCIÓN } \\
\text { AUSPICIANTE (REFERENCIA } \\
\text { BIBLIOGRÁFICA) } \\
\end{array}$ & $\begin{array}{l}\text { OBJETIVOS DECLARADOS EN } \\
\text { CADA INVESTIGACIÓN }\end{array}$ & $\begin{array}{l}\text { METODOLOGÍA UTILIZADA POR } \\
\text { LOS ESTUDIOS RELEVADOS }\end{array}$ & $\begin{array}{l}\text { CORPUS DE ANÁLISIS } \\
\text { DE CADA ESTUDIO }\end{array}$ \\
\hline $\begin{array}{l}\text { Stella Martini y Lila Luchessi, profeso- } \\
\text { ras de la UBA, con alumnos e investi- } \\
\text { gadores de la carrera. } \\
\text { (Martini \& Luchessi, 2004) }\end{array}$ & $\begin{array}{l}\text { Experimentar formas de acceso (mé- } \\
\text { todos) y categorías (teorías) para } \\
\text { interpretar la práctica del periodismo } \\
\text { (p. 184). "Poner en escena las rutinas } \\
\text { productivas a partir de la explicación } \\
\text { de los periodistas"(p. 185). }\end{array}$ & $\begin{array}{l}\text { Observación participante Ilevada a } \\
\text { cabo por estudiantes avanzados de las } \\
\text { cátedras Teorías sobre el periodismo de } \\
\text { la UBA. Etapas (p. 187): } \\
\text { 1. Diseño de investigación. } \\
\text { 2. Muestra y contactos. } \\
\text { 3. Trabajo de campo. } \\
\text { 4. Procesamiento, clasificación y orde- } \\
\text { namiento de categorías. } \\
\text { 5. Interpretación y redación. }\end{array}$ & $\begin{array}{l}45 \text { periodistas de Buenos Aires } \\
\text { Marzo y junio de } 2003 \text {. }\end{array}$ \\
\hline \multicolumn{4}{|l|}{ Año de realización: 2005} \\
\hline \multicolumn{4}{|c|}{ "Sobre los periodistas y su profesión" [FOP05] } \\
\hline $\begin{array}{l}\text { RESPONSABLES / INSTITUCIÓN } \\
\text { AUSPICIANTE (REFERENCIA } \\
\text { BIBLIOGRÁFICA) }\end{array}$ & $\begin{array}{l}\text { OBJETIVOS DECLARADOS EN } \\
\text { CADA INVESTIGACIÓN }\end{array}$ & $\begin{array}{l}\text { METODOLOGÍA UTILIZADA POR } \\
\text { LOS ESTUDIOS RELEVADOS }\end{array}$ & $\begin{array}{l}\text { CORPUS DE ANÁLISIS } \\
\text { DE CADA ESTUDIO }\end{array}$ \\
\hline $\begin{array}{l}\text { Consultora Giacobbe y Asociados para } \\
\text { el Foro de Periodismo Argentino. } \\
\text { (Foro de Periodismo Argentino \& Gia- } \\
\text { cobbe y Asoc., 2005) }\end{array}$ & $\begin{array}{l}\text { Indagar sobre el ejercicio periodístico } \\
\text { en Argentina (problemas de la pro- } \\
\text { fesión, condicionamientos percibidos, } \\
\text { ética profesional, formación académi- } \\
\text { ca; periodistas referenciales). }\end{array}$ & $\begin{array}{l}\text { Cuestionario cerrado, anónimo distri- } \\
\text { buido entre los socios de la organiza- } \\
\text { ción y referencias de esos informantes. }\end{array}$ & $\begin{array}{l}282 \text { periodistas de la ciudad de Buenos } \\
\text { Aires y } 17 \text { provincias del país. La lista se } \\
\text { consolidó a partir del listado de socios } \\
\text { y referidos. } \\
\text { Junio y noviembre de } 2005 \text {. }\end{array}$ \\
\hline
\end{tabular}


Año de realización: 2008

\begin{tabular}{|c|c|c|c|}
\hline \multicolumn{4}{|c|}{ "Periodistas y empresas: claves de una relación necesaria índice" [AUS08] } \\
\hline $\begin{array}{l}\text { RESPONSABLES / INSTITUCIÓN } \\
\text { AUSPICIANTE (REFERENCIA } \\
\text { BIBLIOGRÁFICA) }\end{array}$ & $\begin{array}{l}\text { OBJETIVOS DECLARADOS EN } \\
\text { CADA INVESTIGACIÓN }\end{array}$ & $\begin{array}{l}\text { METODOLOGÍA UTILIZADA POR } \\
\text { LOS ESTUDIOS RELEVADOS }\end{array}$ & \begin{tabular}{|l} 
CORPUS DE ANÁLISIS \\
DE CADA ESTUDIO
\end{tabular} \\
\hline $\begin{array}{l}\text { Lía Ghelfi de la consultora Estudio de } \\
\text { Comunicación y las profesoras Marcela } \\
\text { Pizarro y María José Müller por la Fa- } \\
\text { cultad de comunicación de la Univer- } \\
\text { sidad Austral. } \\
\text { (Estudio de Comunicación \& Universi- } \\
\text { dad Austral, 2008) }\end{array}$ & $\begin{array}{l}\text { Profundizar en la percepción que tie- } \\
\text { nen los periodistas sobre la forma de } \\
\text { comunicarse de las empresas, anali- } \\
\text { zando la valoración de los profesiona- } \\
\text { les de los medios sobre la información } \\
\text { que generan las empresas, los canales } \\
\text { que utilizan, la calidad de los materia- } \\
\text { les informativos que producen, entre } \\
\text { otros aspectos. }\end{array}$ & $\begin{array}{l}\text { Investigación cuantitativa, aplicando } \\
\text { como instrumento de recolección un } \\
\text { cuestionario semi-estructurado (44 } \\
\text { preguntas). Aclara el estudio que no } \\
\text { hay intención de probar o comprobar } \\
\text { ninguna hipótesis en particular, sino de } \\
\text { comprender mejor el vínculo que une } \\
\text { a los periodistas y a los profesionales } \\
\text { de las empresas y qué implicaciones } \\
\text { tiene. }\end{array}$ & $\begin{array}{l}\text { Muestra final de } 174 \text { casos (sobre } 275 \\
\text { contactos telefónicos y correo electró- } \\
\text { nico) de medios de Buenos Aires y los } \\
\text { principales diarios de Córdoba, Men- } \\
\text { doza, Rosario, Mar del Plata, Tucumán, } \\
\text { La Plata y Bahía Blanca. } \\
\text { Selección aleatoria sobre un marco de } \\
\text { muestra conformado por listados de } \\
\text { periodistas y estratificado por tipos de } \\
\text { medios (gráficos, audiovisuales, digi- } \\
\text { tales y agencias de noticias). Se pon- } \\
\text { deró la participación de periodistas por } \\
\text { medio en función de la representativi- } \\
\text { dad de los medios según la influencia } \\
\text { de estos en el escenario público. } \\
\text { Entre enero y marzo de } 2008 \text {. }\end{array}$ \\
\hline \multicolumn{4}{|l|}{ Año de realización: 2009} \\
\hline \multicolumn{4}{|c|}{ "La imagen de los profesionales de la información en la prensa" [UDS09] } \\
\hline $\begin{array}{l}\text { RESPONSABLES / INSTITUCIÓN } \\
\text { AUSPICIANTE (REFERENCIA } \\
\text { BIBLIOGRÁFICA) }\end{array}$ & $\begin{array}{l}\text { OBJETIVOS DECLARADOS EN } \\
\text { CADA INVESTIGACIÓN }\end{array}$ & $\begin{array}{l}\text { METODOLOGÍA UTILIZADA POR } \\
\text { LOS ESTUDIOS RELEVADOS }\end{array}$ & $\begin{array}{l}\text { CORPUS DE ANÁLISIS } \\
\text { DE CADA ESTUDIO }\end{array}$ \\
\hline $\begin{array}{l}\text { Graciela Paredes, Universidad del Sal- } \\
\text { vador, Fac. Cs. Educ.y de Com. Social. } \\
\text { (Paredes, [2010]) }\end{array}$ & $\begin{array}{l}\text { A partir del análisis de noticias sobre } \\
\text { periodistas, se intentaba recabar qué } \\
\text { definición daban de la profesión los } \\
\text { periodistas participantes en las notas; } \\
\text { los rasgos con los que debían contar } \\
\text { los periodistas; su función social; con- } \\
\text { dicionamientos a la labor periodística. }\end{array}$ & $\begin{array}{l}\text { Monitoreo de noticias referidas a la } \\
\text { temática del campo periodístico en los } \\
\text { siete diarios de Buenos Aires durante } \\
2009 \text {. }\end{array}$ & $\begin{array}{l}2415 \text { noticias cuyos protagonistas son } \\
\text { periodistas o propietarios de medios. } \\
\text { Universo: } 5.041 \text { unidades redacciona- } \\
\text { les en diarios de Buenos Aires publica- } \\
\text { dos entre enero y diciembre de } 2009 \text {. }\end{array}$ \\
\hline \multicolumn{4}{|l|}{ Año de realización: 2011} \\
\hline \multicolumn{4}{|c|}{ “Encuesta sobre los periodistas y su profesión” [F0P11] } \\
\hline \begin{tabular}{|l} 
RESPONSABLES / INSTITUCIÓN \\
AUSPICIANTE (REFERENCIA \\
BIBLIOGRÁFICA) \\
\end{tabular} & $\begin{array}{l}\text { OBJETIVOS DECLARADOS EN } \\
\text { CADA INVESTIGACIÓN }\end{array}$ & $\begin{array}{l}\text { METODOLOGÍA UTILIZADA POR } \\
\text { LOS ESTUDIOS RELEVADOS }\end{array}$ & \begin{tabular}{|l} 
CORPUS DE ANÁLISIS \\
DE CADA ESTUDIO
\end{tabular} \\
\hline $\begin{array}{l}\text { Cecilia Mosto, responsable por la con- } \\
\text { sultora Cl0 para el Foro de periodismo } \\
\text { argentino. } \\
\text { (Foro de Periodismo Argentino \& CIO } \\
\text { Argentina, 2011) }\end{array}$ & $\begin{array}{l}\text { Conocer la opinión de los periodistas } \\
\text { argentinos sobre distintos aspectos de } \\
\text { su profesión y del entorno mediato } \\
\text { e inmediato dentro del cual la desa- } \\
\text { rrollan. } \\
\text { - Principales problemas } \\
\text {-Ética y rigor profesional } \\
\text { - Motivaciones profesionales } \\
\text { - Libertad periodística } \\
\text { - Capacitación } \\
\text { - Expectativas. Referentes de la pro- } \\
\text { fesión } \\
\text { - Lugar y modalidad de trabajo } \\
\text { - Asuntos gremiales. }\end{array}$ & $\begin{array}{l}\text { Selección de contactos aleatorios siste- } \\
\text { máticos a partir de un listado provisto } \\
\text { por la organización a través de sus so- } \\
\text { cios de todo el país. Encuesta anónima } \\
\text { en página web (formato (AWI), con } \\
\text { cuestionario semi-estructurado (50 } \\
\text { preguntas cerradas y abiertas). }\end{array}$ & $\begin{array}{l}943 \text { casos en todo el país en base a } \\
\text { listados de periodistas. } \\
\text { Junio a julio } 2011\end{array}$ \\
\hline
\end{tabular}

